\title{
Mechanical and Electrical Monitoring in the Dynamics of Twisted Phosphorene Nanoflakes on 2D Monolayers
}

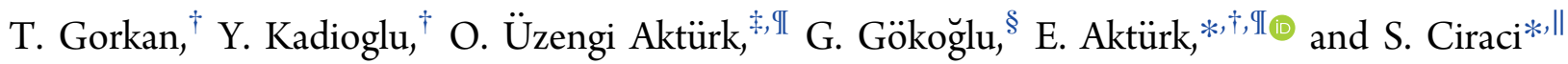 \\ ${ }^{\dagger}$ Department of Physics, Adnan Menderes University, Aydın 09010, Turkey \\ ${ }^{*}$ Department of Electrical \& Electronics Engineering, Adnan Menderes University, Aydın 09010, Turkey \\ ${ }^{\mathbb{I}}$ Nanotechnology Application and Research Center, Adnan Menderes University, Aydın 09010, Turkey \\ ${ }^{\S}$ Department of Mechatronics Engineering, Faculty of Engineering, Karabuk University, 78050 Karabuk, Turkey \\ "Department of Physics, Bilkent University, Ankara 06800, Turkey
}

\begin{abstract}
We investigated the rotational and translational dynamics of hydrogen-passivated, black phosphorene and blue phosphorene nanoflakes of diverse size and geometry anchored to graphene, black phosphorene, blue phosphorene, and $\mathrm{MoS}_{2}$ monolayer substrates. The optimized attractive interaction energy between each nanoflake and monolayer substrates are harmonic for small angular displacements, leading to libration frequencies. We showed that the relevant dynamical parameters and resulting libration frequencies, which vary with the size/geometry of nanoflakes, as well as with the type of substrate, can be monitored by charging, external electric field, pressure, and also by a molecule anchored to the flake. The optimized energy profiles and energy barriers thereof have been calculated in translational and in large angle rotational dynamics. Owing to the weak interaction between the flakes and monolayers the energy barriers are particularly small for incommensurate systems and can renders nearly frictionless rotation and translation, which is crucial for nanoscale mechanics. Even if small for particular combined nanoflake + monolayer heterostructures, the energy band gaps exhibit variations with angular and linear displacements of nanoflakes. However, these band gaps undergo considerable reduction under pressure. With tunable dynamics, electronic structure, and low friction coefficients, individual or periodically repeating nanoflakes on a monolayer substrate constitute critical composite structures offering the design of novel detectors, nanomechanical, electromechanical, and electronic devices.
\end{abstract}

\section{INTRODUCTION}

Free-standing, two-dimensional (2D) materials, like graphene, h-BN, and $\mathrm{MoS}_{2}$, have been introduced having very strong, inplane covalent bonding which provides the stability of the unsupported sheet (flake) structures with one-atom or a few atoms thick layers. van der Waals (vdW) heterostructures, constructed by the vertical stacking of these $2 \mathrm{D}$ monolayers, have attracted considerable attention recently, due to immense novel material options with critical functionalities. ${ }^{1}$ Some of the heterostructures constructed by stacking different $2 \mathrm{D}$ sheets on top of each other have shown peculiar properties, which are absent in isolated monolayers or in parent layered structures. ${ }^{2}$ These systems have been fabricated in the past decade using molecular beam epitaxy methods and some systems have been studied revealing intriguing properties observed in nanoscale. $^{3-12}$ While various artificial materials have been constructed by using 2D layers having different properties, ${ }^{13}$ a nanoflake of a $2 \mathrm{D}$ monolayer situated on another extended 2D monolayer can be considered as the simplest heterostructure. Even if commensurability or lattice mismatch between vertical stacked layers do not hinder the growth of material owing to the weak vdW attraction, the crystalline alignment between the layers at the atomic scale can be critical for the electronic coupling and for the resulting device characteristics. ${ }^{14}$

On the other side, molecules, atomic clusters even nanoflakes have been widely used to modify electronic properties of 2D materials providing functionalization. ${ }^{15-21}$ In addition to electronic structure modification, the molecules or nanoflakes can also behave as nanomechanical devices on the surfaces. The design and synthesis of molecular nanomachines have also been reported. ${ }^{22,23}$ The nanometer-size flakes of 2D materials on 2D sheets, like graphene flake on graphene ${ }^{24,25}$ display crucial dynamical behaviors. It was shown that the translational and rotational displacements of the flakes on graphene surface can generate restoring forces which can lead to a harmonic motion with a characteristic frequency. Additionally, this frequency is also dependent on the shape and size of the flake. ${ }^{25}$ Recently, the direct imaging of rotating molecules on single-layer graphene sheet was

Received: September 26, 2019

Revised: November 14, 2019

Published: November 25, 2019 
monitored experimentally. ${ }^{26,27}$ It was observed that the rotation of graphene on h-BN can thermally be induced at a temperature of $T>100{ }^{\circ} \mathrm{C} .{ }^{26}$ The interlayer van der Waals interaction also depends on relative orientation of $2 \mathrm{D}$ layers. These observations show that flakes or molecules can easily be rotated or translated on sheets due to weak van der Waals forces and the small energy barriers can be overcome by small excitations. It can also be concluded that the slowly varying energy in translational motion corresponds to low frictional force. Superlubricity is one of the most interesting phenomenon exhibited by graphene, wherein friction forces between the incommensurate graphene layers is very low. ${ }^{6,28-30}$ Although vdW interaction is evident in many heterostructure systems, as an exceptional case, graphene flake can also form covalent type bonding on a Si-terminated $\mathrm{SiC}$ substrate at suitable rotation angles. ${ }^{31}$

The dynamic behavior of the individual molecules on surfaces have been probed experimentally; these studies contributed significantly toward a better understanding of the structural dynamics of complex systems. ${ }^{32,33}$ The motion of molecules and flakes can be rotational or translational as well as librational under the optical and thermal excitations and any other external forces. This knowledge forms a basis for the design and realization of nanomechanical devices.

In this study, we present number of critical properties revealed from our extensive analysis of the rotational and translational dynamics of the edge-hydrogenated black phosphorene $(\alpha$-PNFH) and blue phosphorene nanoflakes $(\beta$-PNFH), on the surface of $2 \mathrm{D}$ graphene, black phosphorene $(\alpha-\mathrm{P})$, blue phosphorene $(\beta-\mathrm{P})$, and $\mathrm{MoS}_{2}$ monolayers. Phosphorene nanoflakes have been considered in various sizes with coronene, triangular, and parallelogram shapes with both zigzag and armchair edges. All angular force constants and angular frequencies upon librational motion on the surfaces have been calculated. As a proof of concept, we also consider the effect of glycine molecule, a nonpolar and well-known amino acid, anchored to the flakes on a specific monolayer. Similarly, the effects of charging, applied electric field and compressive force on rotational dynamics of the flakes has also been examined. Rotational and translational energy barriers have been estimated and their dependency on flake type, size, and shape are clarified. The angular/libration frequencies of flakes on substrates are largely altered by the flake size and type of substrate and also by a glycine molecule placed on a flake. We showed that the rotational dynamics of a flake on various substrate monolayers can easily be tuned by charging, adsorbed molecules, and electric fields, etc. As a proof of concept, we also demonstrate that an external force on a specific flake can induce dramatic change in the band gap. This analysis reveals the effect of various parameters to control the dynamics of flakes on $2 \mathrm{D}$ sheets and can provide necessary background to design novel electromechanical nanoscale systems/detectors for specific purposes.

\section{COMPUTATIONAL DETAILS}

All the calculations relevant to total energy and rotational/ transitional motions of the flakes on the substrates are performed within density functional theory (DFT) by using plane-wave basis set and projector augmented wave (PAW) potentials as implemented in the Vienna ab initio simulation package, VASP. ${ }^{34,35}$ The exchange-correlation potentials are represented by the generalized gradient approximation (GGA) with the Perdew-Burke-Ernzerhof $(\mathrm{PBE})^{36}$ exchange corre- lation functionals including the van der Waals (vdW) correction in DFT-D2 level. ${ }^{37}$ In order to avoid flake-flake interactions between the adjacent cells, we study with $12 \times 12$, $9 \times 6,10 \times 10$, and $7 \times 7$ supercells for graphene, black phosphorene, blue phosphorene, and $\mathrm{MoS}_{2}$ substrates, respectively. These meshes provide at least $10 \AA$ lateral distance between the flakes on the substrate resulting in negligible coupling. A vacuum distance along the $z$-direction is taken as $15 \AA$. The energy convergence value between two consecutive steps is chosen as $10^{-5} \mathrm{eV}$, which ensures a wellconverged ground state. Kinetic energy cutoff $\hbar^{2}|\mathbf{k}+\mathbf{G}|^{2} / 2 m$ for the plane-wave basis set is taken as $450 \mathrm{eV}$. Brillouin zone integration is performed by a $3 \times 3 \times 1 k$-point grid. These values regarding energy cutoff and $k$-mesh have been checked for convergence. The ionic minimization process is performed by conjugate gradient (CG) method in which force on each atom is reduced to less than $0.001 \mathrm{eV} / \AA$. Gaussian broadening on Fermi level is used with a smearing width $0.01 \mathrm{eV}$. In order to obtain libration frequencies under an external electric field, we use $a b$ initio DFT as implemented in the SIESTA code, ${ }^{38}$ which utilize local basis sets in terms of numerical orbitals, and is suitable to treat charged systems.

The average cohesive energy (per atom) of $\mathrm{H}$ saturated nanoflake is calculated as $E_{c}=\left(E_{P N F H}-n_{P} E_{P}-n_{H} E_{H}\right) /\left(n_{P}+\right.$ $\left.n_{H}\right)$ where $E_{P N F H}$ is the calculated total energy of a given edgepassivated nanoflake saturated by $\mathrm{H}$ atoms, $E_{P}$ is the calculated total energy of an isolated $\mathrm{P}$ atom, and $E_{H}$ is that of the isolated hydrogen atom, $n_{P}$ and $n_{H}$ are the total number of $\mathrm{P}$ and $\mathrm{H}$ atoms of the nanoflake, respectively.

\section{SMALL ANGLE ROTATIONAL DYNAMICS OF NANOFLAKES ON SUBSTRATES: LIBRATION FREQUENCY}

We consider $\alpha$-PNFHs on graphene, $\alpha$-P, and $\mathrm{MoS}_{2}$ monolayer substrates and $\beta$-PNFHs on graphene, $\beta$-P, and $\mathrm{MoS}_{2}$ monolayer substrates. All flakes considered in this study have been edge-passivated with hydrogen to hinder edge reconstruction, to prevent edge atoms from strong chemical interaction with substrate, to strengthen thermal and structural stability. Nanoflakes of diverse size and shape, namely triangular $(\mathrm{t})$, coronene $(\mathrm{c})$, and parallelogram $(\mathrm{p})$ shapes and both zigzag (zz) and armchair (ac) edge geometries are considered. The size of the flakes is varied from $13 \mathrm{P}$ atoms to $36 \mathrm{P}$ atoms with varying $\mathrm{H}$ atoms (e.g., $13 \mathrm{P}+9 \mathrm{H}, 36 \mathrm{P}+18 \mathrm{H}$ ). All the edge-passivated nanoflakes considered in this study have been found to be stable as a stand-alone molecule. In our notation, c-zz $/ 24 \mathrm{P}+12 \mathrm{H}$ stands for a coronene nanoflake with zigzag edges consisting of $24 \mathrm{P}$ and $12 \mathrm{H}$ atoms. In Figure 1, we illustrate the top views of the atomic structures of edgepassivated, diverse phosphorene nanoflakes considered in this study. In the same figure, we present also their calculated average binding energies and HOMO-LUMO gaps.

When a nanoflake is placed on a monolayer, it is anchored to a well-defined equilibrium position. We display the selected equilibrium structures and stacking configurations of diverse flake + substrate systems denoted as $\mathrm{AA}, \mathrm{AB}$, and $\mathrm{AC}$ in Figure 2 for $\mathrm{c}-\mathrm{zz} / 24 \mathrm{P}+12 \mathrm{H}$ type nanoflakes. In Figure 3, we show the calculated variation of the interaction energy between two specific flakes anchored to different monolayers with the rotation angle $\theta$. Each configuration corresponds to a height $z$ leading to minimum energy. As depicted in Figure 3, a restoring force appears on the flake resulting from the twisting behavior on substrate like a torsional pendulum. A flake tends 

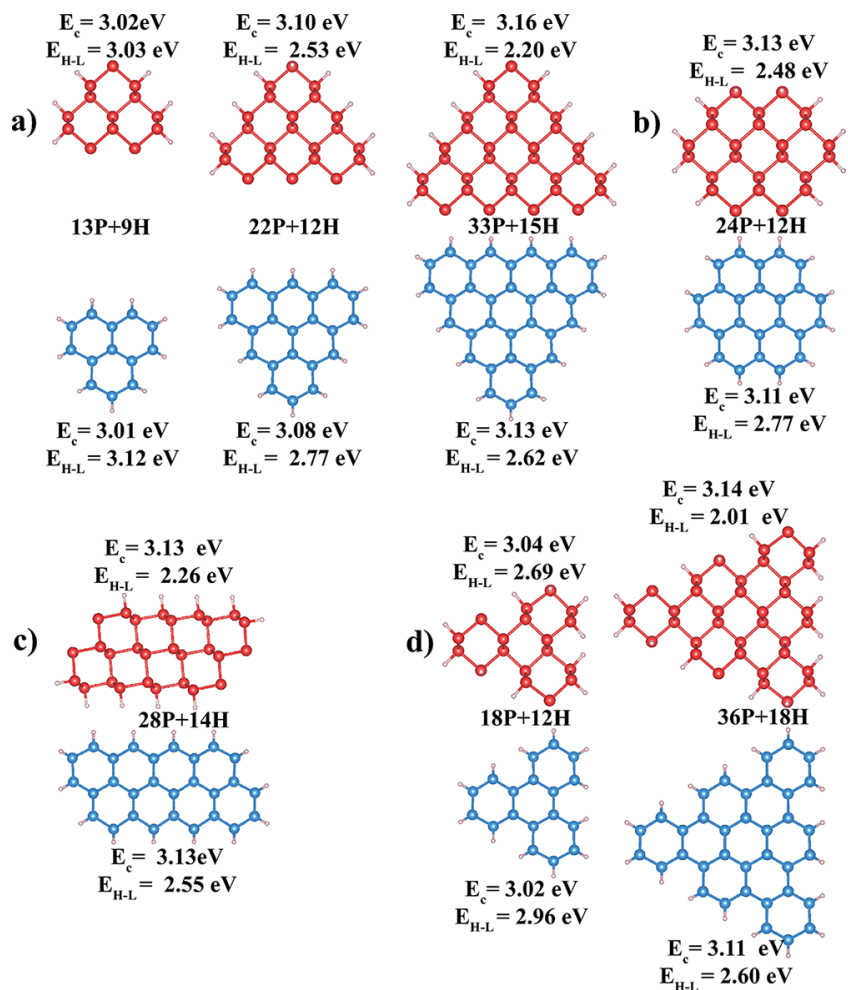

Figure 1. Top views of optimized atomic configurations of hydrogensaturated, black, $\alpha$-PNFH, and blue, $\beta$-PNFH, phosphorene nanoflakes, which are considered in this study. Their calculated, average cohesive energies per atom $\left(E_{c}\right)$ and HOMO-LUMO gaps $\left(E_{H-L}\right)$ are also presented. Key: (a) Free-standing, triangular-zigzag ( $\mathrm{t}$-zz); (b) coronene zigzag (c-zz); (c) parallelogram-zigzag (p-zz); and (d) triangular-armchair ( $\mathrm{t}-\mathrm{ac})$. Atoms of $\alpha$-PNFH and $\beta$-PNFH are represented by red and blue balls, respectively. Small balls indicate saturating hydrogen atoms.

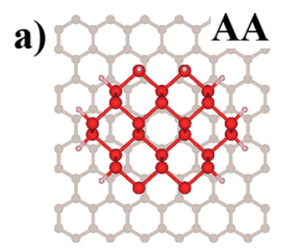

b)

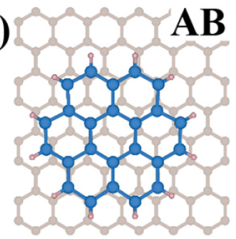

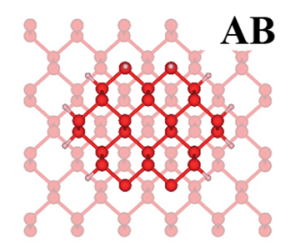

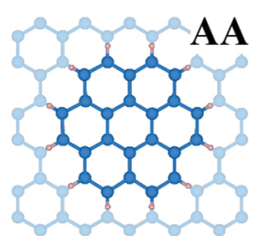

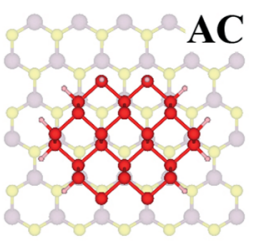

AA

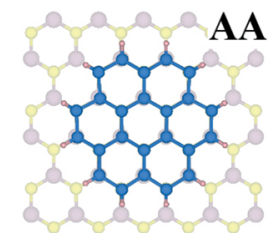

Figure 2. Selected equilibrium structures and stacking configurations of a specific flake on diverse substrates. (a) $\alpha$-PNFH flake of c-zz/24P $+12 \mathrm{H}$ type on graphene, $\alpha-\mathrm{P}$, and $\mathrm{MoS}_{2}$ monolayers. (b) $\beta$-PNFH flake of c-zz/24P+12H type on graphene, $\beta$-P, and $\mathrm{MoS}_{2}$ monolayers.

to return to its equilibrium position, when a small angular displacement was applied. Accordingly, the flake executes a simple harmonic motion around its equilibrium position under a harmonic interaction energy. ${ }^{25}$

In this section, we examine the small angle displacements around the equilibrium and reveal the libration frequency $f$ thereof. The dynamics related with large angle displacements will be treated in forthcoming sections. Moment of inertia values, $I=\sum_{i} m_{i} r_{i}^{2}$, have been calculated due to a rotation axes
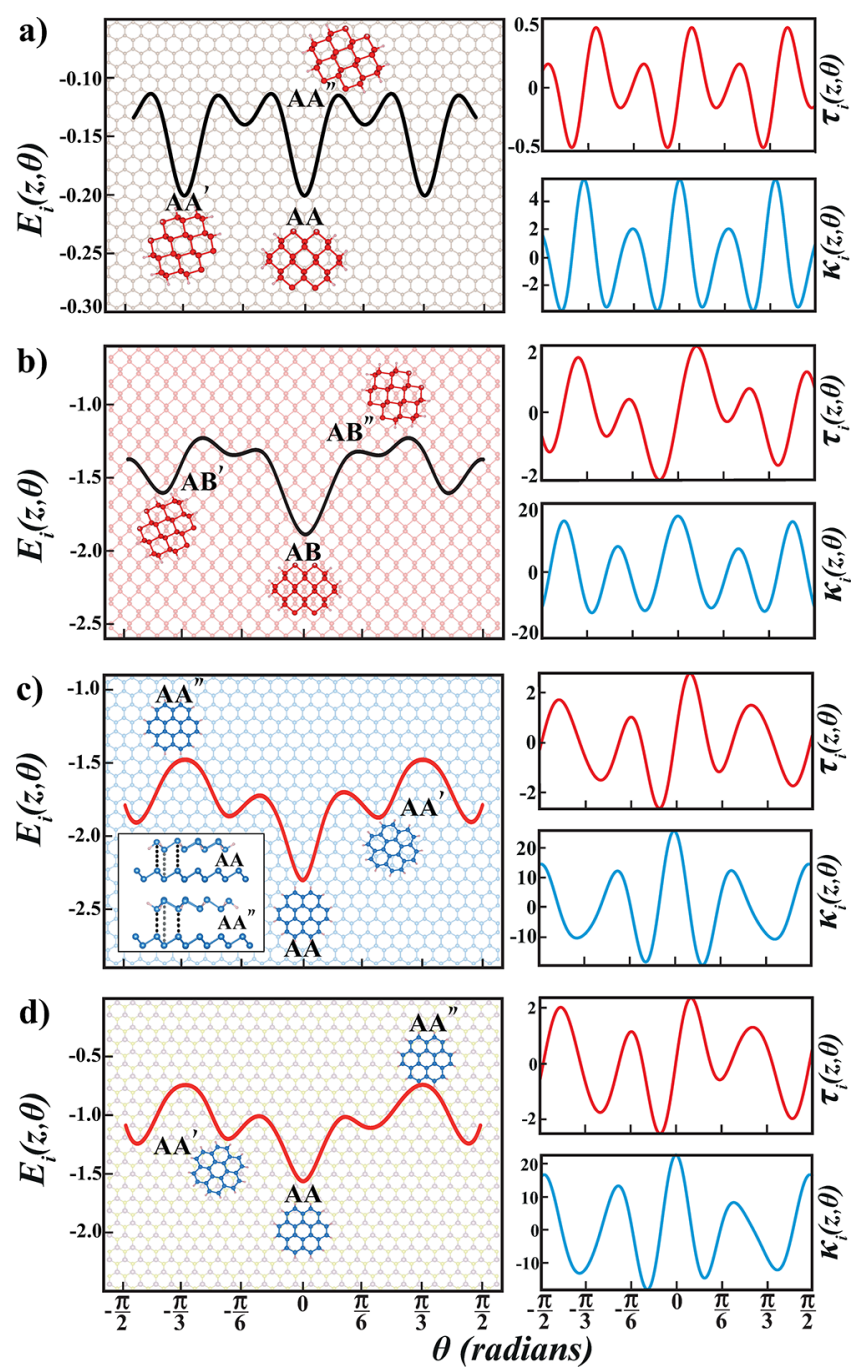

Figure 3. Calculated variations of the interaction energy, $E_{i}(z, \theta)$, torque, $\tau(z, \theta)$, and rotational force constant, $\kappa(z, \theta)$, with the rotational angle, $\theta$, for $c-z z / 24 \mathrm{P}+12 \mathrm{H}$ type flakes on different substrates: $\alpha$-PNFH on graphene (a) and on $\alpha$-P monolayer (b). $\beta$ PNFH on $\beta$-P (c) and on $\mathrm{MoS}_{2}$ monolayer(d). The interaction energies are optimized relative to the height $z$ between the flake and monolayer for each $\theta$. Notably, while specific equilibrium sites correspond to global minima, others are metastable.

which passes through center of mass of the flake perpendicular to plane. The position of the rotation axis in equilibrium is mainly determined through the interaction between flake and substrate, and hence it may be subject to very minute translations in small angle rotations around the minimum energy configurations described in Figure 3. Therefore, a given flake with definite shape and size has a unique $I$. As one expects, $I$ increases with the increasing size of the flake. Rotational force constants, $\kappa$, and angular, $\omega$, or libration, $f$, frequencies can be calculated by using the formulas, $\kappa=\partial^{2} E_{i} /$ $\partial \theta^{2}$, and $\omega=2 \pi f$, where $f=\sqrt{\kappa / I}$, respectively. In Table 1 , we show the calculated rotational parameters of the PNFHs: i.e., rotational force constants, $\kappa$, and rotational vibration frequencies, $\omega$, as well as equilibrium stacking configuration on the related substrates.

The equilibrium state depends on the flake size as seen in Table 1, so that different stacking configurations can be energetically favored for different flakes. The variations of the 
Table 1. Equilibrium Stacking Configurations and the Parameters of the Rotational Dynamics of Edge-Passivated Phosphorene Nanoflakes on Various Monolayer Substrates ${ }^{a}$

\begin{tabular}{|c|c|c|c|c|c|c|c|c|}
\hline \multirow[b]{2}{*}{ type } & \multicolumn{4}{|c|}{$\beta$-PNFH } & \multicolumn{4}{|c|}{$\alpha$-PNFH } \\
\hline & subs. & site & $\kappa\left(\times 10^{-18}\right)$ & $\omega\left(\times 10^{12}\right)$ & subs. & site & $\kappa\left(\times 10^{-18}\right)$ & $\omega\left(\times 10^{12}\right)$ \\
\hline $\mathrm{t}-\mathrm{zz} / 13 \mathrm{P}+9 \mathrm{H}$ & grap. & $\mathrm{AB}$ & 0.56 & 2.93 & grap. & $\mathrm{AB}$ & 0.22 & 1.99 \\
\hline $\mathrm{t}-\mathrm{ac} / 18 \mathrm{P}+12 \mathrm{H}$ & grap. & $\mathrm{AA}$ & 0.76 & 2.33 & grap. & $\mathrm{AA}$ & 0.50 & 2.07 \\
\hline $\mathrm{t}-\mathrm{zz} / 22 \mathrm{P}+12 \mathrm{H}$ & grap. & AA & 0.34 & 1.31 & grap. & $\mathrm{AA}$ & 0.83 & 2.24 \\
\hline$c-z z / 24 \mathrm{P}+12 \mathrm{H}$ & grap. & $\mathrm{AB}$ & 0.46 & 1.44 & grap. & $\mathrm{AA}$ & 0.90 & 2.21 \\
\hline $\mathrm{p}-\mathrm{zz} / 28 \mathrm{P}+14 \mathrm{H}$ & grap. & AA & 0.68 & 1.34 & grap. & $\mathrm{AA}$ & 1.25 & 2.03 \\
\hline $\mathrm{t}-\mathrm{zz} / 33 \mathrm{P}+15 \mathrm{H}$ & grap. & $\mathrm{AA}$ & 0.67 & 1.20 & grap. & $\mathrm{AA}$ & 1.53 & 1.99 \\
\hline $\mathrm{t}-\mathrm{ac} / 36 \mathrm{P}+18 \mathrm{H}$ & grap. & $\mathrm{AB}$ & 0.85 & 1.24 & grap. & $\mathrm{AA}$ & 2.07 & 2.06 \\
\hline $\mathrm{t}-\mathrm{zz} / 13 \mathrm{P}+9 \mathrm{H}$ & $\beta-\mathrm{P}$ & $\mathrm{AB}$ & 1.66 & 5.04 & $\alpha-\mathrm{P}$ & $\mathrm{AB}$ & 0.51 & 3.04 \\
\hline $\mathrm{t}-\mathrm{ac} / 18 \mathrm{P}+12 \mathrm{H}$ & $\beta-\mathrm{P}$ & $\mathrm{AB}$ & 4.05 & 5.37 & $\alpha-\mathrm{P}$ & $\mathrm{AB}$ & 1.83 & 3.96 \\
\hline $\mathrm{t}-\mathrm{zz} / 22 \mathrm{P}+12 \mathrm{H}$ & $\beta-\mathrm{P}$ & $\mathrm{AB}$ & 7.66 & 6.23 & $\alpha-\mathrm{P}$ & $\mathrm{AB}$ & 2.43 & 3.83 \\
\hline$c-z z / 24 \mathrm{P}+12 \mathrm{H}$ & $\beta-\mathrm{P}$ & $\mathrm{AA}$ & 4.63 & 4.58 & $\alpha-\mathrm{P}$ & $\mathrm{AB}$ & 2.84 & 3.92 \\
\hline $\mathrm{p}-\mathrm{zz} / 28 \mathrm{P}+14 \mathrm{H}$ & $\beta-\mathrm{P}$ & $\mathrm{AA}$ & 8.43 & 4.73 & $\alpha-\mathrm{P}$ & $\mathrm{AB}$ & 3.57 & 3.43 \\
\hline $\mathrm{t}-\mathrm{zz} / 33 \mathrm{P}+15 \mathrm{H}$ & $\beta-\mathrm{P}$ & $\mathrm{AA}$ & 10.19 & 4.70 & $\alpha-\mathrm{P}$ & $\mathrm{AB}$ & 4.24 & 3.32 \\
\hline $\mathrm{t}-\mathrm{ac} / 36 \mathrm{P}+18 \mathrm{H}$ & $\beta-\mathrm{P}$ & $\mathrm{AA}$ & 13.53 & 4.94 & $\alpha-\mathrm{P}$ & $\mathrm{AB}$ & 6.96 & 3.78 \\
\hline$c-z z / 24 \mathrm{P}+12 \mathrm{H}$ & $\mathrm{MoS}_{2}$ & AA & 3.90 & 4.21 & $\mathrm{MoS}_{2}$ & $\mathrm{AC}$ & 2.07 & 3.34 \\
\hline $\mathrm{t}-\mathrm{zz} / 33 \mathrm{P}+15 \mathrm{H}$ & $\mathrm{MoS}_{2}$ & AA & 7.02 & 3.90 & $\mathrm{MoS}_{2}$ & $\mathrm{AC}$ & 3.46 & 2.99 \\
\hline
\end{tabular}

${ }^{a_{T}}$ Type of $\alpha$-PNFHs and $\beta$-PNFHs; types of monolayer substrate, Subs; equilibrium site; angular force constant $\kappa$ in $\left(\mathrm{eV} / \mathrm{rad}^{2}\right)$; angular frequency $\omega$ in $(\mathrm{rad} / \mathrm{s})$. As an example, $\mathrm{t}-\mathrm{zz} / 13 \mathrm{P}+9 \mathrm{H}$ indicates a zigzag edged and $\mathrm{H}$ passivated triangular flake comprising $13 \mathrm{P}$ and $9 \mathrm{H}$ atoms.

Table 2. Angular Force Constant, $\kappa\left(\mathrm{eV} / \mathrm{rad}^{2}\right)$ and Angular Frequency, $\omega(\mathrm{rad} / \mathrm{s})$ Values of Selected Edge-Passivated Phosphorene Nanoflakes $\beta$-PNFH and $\alpha$-PNFH on Various Monolayer Substrates Calculated for an Excess Charge of -5 Electrons, for a Single Glycine Molecule Attached to the Nanoflake, or for an Electric Field of 0.5 V/Å Applied Perpendicular to the Flake + Substrate System ${ }^{a}$

\begin{tabular}{|c|c|c|c|c|c|c|c|}
\hline & \multirow[b]{2}{*}{ type } & \multicolumn{3}{|c|}{$\beta$-PNFH } & \multicolumn{3}{|c|}{$\alpha$-PNFH } \\
\hline & & subs. & $\kappa\left(\times 10^{-18}\right)$ & $\omega\left(\times 10^{12}\right)$ & subs. & $\kappa\left(\times 10^{-18}\right)$ & $\omega\left(\times 10^{12}\right)$ \\
\hline \multirow[t]{3}{*}{ charge } & $\mathrm{c}-\mathrm{zz} / 24 \mathrm{P}+12 \mathrm{H}$ & grap. & $0.64(0.18)$ & $1.70(0.26)$ & grap & $0.90(0.00)$ & $2.21(0.00)$ \\
\hline & & $\beta-\mathrm{P}$ & $4.78(0.15)$ & $4.66(0.07)$ & $\alpha-\mathrm{P}$ & $2.53(-0.31)$ & $3.70(-0.22)$ \\
\hline & & $\mathrm{MoS}_{2}$ & $3.59(-0.31)$ & $4.04(-0.17)$ & $\mathrm{MoS}_{2}$ & $1.78(-0.29)$ & $3.10(-0.24)$ \\
\hline \multirow[t]{3}{*}{ glycine } & $\mathrm{t}-\mathrm{zz} / 33 \mathrm{P}+15 \mathrm{H}$ & grap. & $0.60(-0.07)$ & $1.14(-0.07)$ & grap & $1.59(0.06)$ & $2.02(0.03)$ \\
\hline & & $\beta-\mathrm{P}$ & $10.28(0.09)$ & $4.71(0.01)$ & $\alpha-\mathrm{P}$ & $4.44(0.20)$ & $3.38(0.06)$ \\
\hline & & $\mathrm{MoS}_{2}$ & $6.89(-0.13)$ & $3.85(-0.04)$ & $\mathrm{MoS}_{2}$ & $3.41(-0.05)$ & $2.96(-0.03)$ \\
\hline \multirow[t]{3}{*}{ electric field } & $c-z z / 24 \mathrm{P}+12 \mathrm{H}$ & grap. & $1.40(0.94)$ & $2.52(1.08)$ & grap & $1.07(0.17)$ & $2.41(0.20)$ \\
\hline & & $\beta$-P & $4.12(-0.51)$ & $4.32(-0.26)$ & $\alpha-\mathrm{P}$ & $2.06(-0.78)$ & $3.34(-0.58)$ \\
\hline & & $\mathrm{MoS}_{2}$ & $3.56(-0.34)$ & $4.02(-0.19)$ & $\mathrm{MoS}_{2}$ & $3.49(1.42)$ & $4.34(1.00)$ \\
\hline
\end{tabular}

${ }^{a_{T}}$ The changes in the values of $\kappa$ and $\omega$ relative to original bare values are given in parentheses. Negative values in parentheses indicate a decrease.

rotational frequencies of nanoflakes on specific monolayers are complex and depend on various parameters. First of all, they vary depending on the flake size and geometry, as well as on the monolayer substrates. These frequencies are approximately comparable to those frequencies of molecular vibrations (e.g., those of glycine ${ }^{39}$ ) and can easily be accessible; they can be excited and detected by infrared and Raman spectroscopy techniques as well as terahertz acoustic waves. In this respect, variation of libration frequency of a flake + monolayer system under external effects may allow us to design nanomechanical detectors.

For the $\alpha$-PNFH + graphene system, $\kappa$ values increase monotonically by flake size of the same geometry. However, angular frequency can decrease for specific geometries even if $\kappa$ increases. A maximum angular frequency of $2.24 \times 10^{12} \mathrm{rad} / \mathrm{s}$, or corresponding libration frequency of $0.71 \mathrm{THz}$ was observed for $\mathrm{t}-\mathrm{zz} / 22 \mathrm{P}+12 \mathrm{H}$ flake, which is smaller than that of $\mathrm{t}-\mathrm{zz} / 33 \mathrm{P}+18 \mathrm{H}$ flake. Similar situation can occur for $\alpha$ $\mathrm{PNFH}$ on $\alpha$-P monolayer. In that system, the rotational force constant, $\kappa$, is three or four times larger than that of $\alpha$-PNFH on graphene monolayer resulting in higher libration frequencies. $\alpha$-PNFH of t-ac/18P $+12 \mathrm{H}$ type has the largest rotational frequency as $3.96 \times 10^{12} \mathrm{rad} / \mathrm{s}$ (or libration frequency of $1.26 \mathrm{THz}$ ) on $\alpha$-P surface.

$\beta$-PNFHs exhibit an irregular trend both on graphene and on $\beta$-P sheet. Both $\kappa$ and $\omega$ quantities show nonmonotonic behavior on both surfaces. The frequencies of $\beta$-PNFHs on $\beta$-P surface are the largest one among the systems considered in this work and are distributed between $4.58 \times 10^{12}$ and $6.23 \times$ $10^{12} \mathrm{rad} / \mathrm{s}$. However, $\beta$-PNFHs on graphene have the lowest frequencies attained. As an example, $\beta$-PNFH of t-zz $/ 22 \mathrm{p}+12 \mathrm{H}$ type has $1.31 \times 10^{12}$ and $6.23 \times 10^{12} \mathrm{rad} / \mathrm{s}$ frequencies on graphene and $\beta$-P monolayers, respectively. It is concluded that the libration frequencies of $\beta$-PNFHs can dramatically be altered on different substrates.

$\mathrm{MoS}_{2}$ monolayer provides a moderate interaction medium for both $\alpha$ - and $\beta$-PNFHs. Angular and hence libration frequencies of $\alpha$ - and $\beta$-PNFHs on the substrates show the following trend like: $\omega_{\text {graphene }}<\omega_{\mathrm{MoS}_{2}}<\omega_{\mathrm{P}}$. Angular force constant, $\kappa$ values are also ordered in the same way. This trend is in compliance with the trend in the binding energies of $\beta$ - 

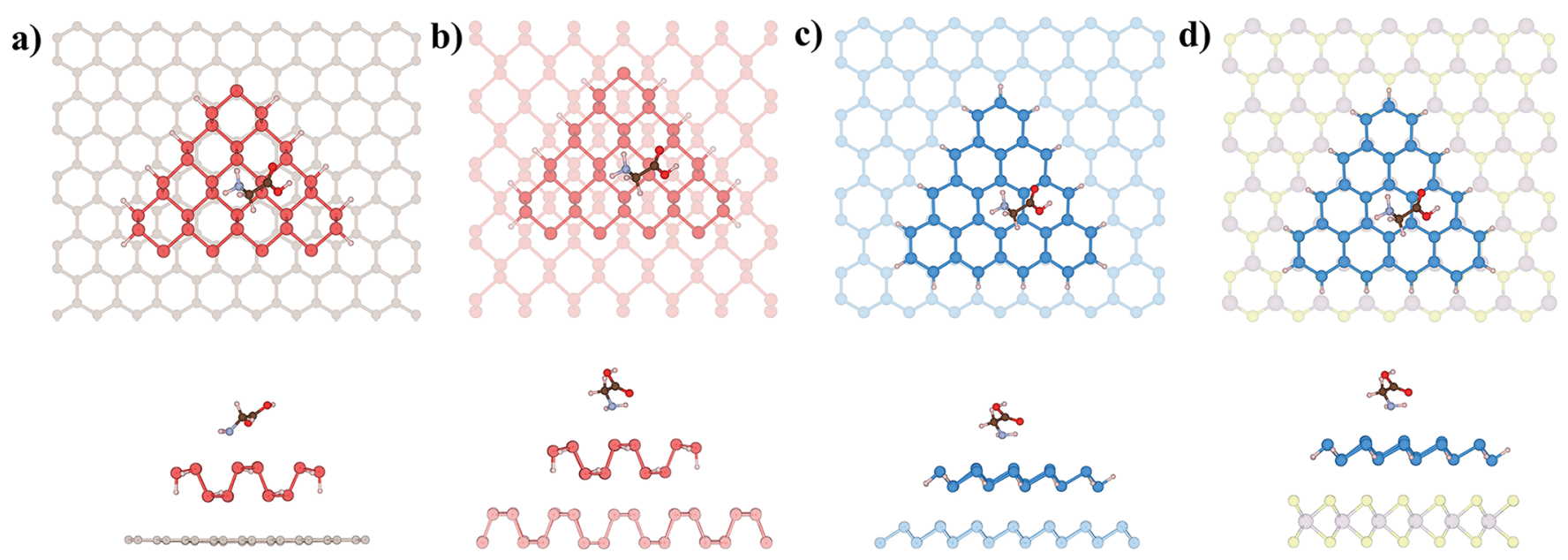

Figure 4. Top and side views of glycine-adsorbed $\alpha$ - and $\beta$-PNFHs on related 2D monolayer substrates. Key: (a) glycine on $\alpha$-PNFH + graphene, (b) glycine on $\alpha$-PNFH $+\alpha$-P, (c) glycine on $\beta$-PNFH $+\beta$-P, and (d) glycine on $\beta$-PNFH $+\mathrm{MoS}_{2}$.

PNFHs on graphene, $\mathrm{MoS}_{2}$ and $\beta$-P surfaces. But, it is not easy to identify a relationship between binding energy and rotational frequency, since the rotational force constant is proportional to the second partial derivative of energy versus $\theta$.

Effect of Charging. The existence of excess charges in the system can modify the distribution of the electronic charge, and hence, they can change the interaction between the flake and the substrate. In Table 2, we present the angular force constants and libration frequencies of a representative flake, $\mathrm{c}$ $\mathrm{zz} / 24 \mathrm{P}+12 \mathrm{H}$, on diverse monolayers under an excess charge of -5 electrons. We see that the rotational (hence libration) frequency of $\alpha$-PNFH on graphene does not change upon charging, while the frequency values of $\alpha$-PNFH on $\alpha$-P decrease with increasing negative excess charge. However, the frequency of $\beta$-PNFH increases on both graphene and $\beta$-P monolayers. Whereas, the rotational frequencies of both $\alpha$ - and $\beta$-PNFHs on $\mathrm{MoS}_{2}$ monolayer decrease with excess electronic charge. This contrasting variations of the libration frequencies for two different substrates are closely related with their electronic structure and flakes, so that different occupancies of electrons give rise to different interactions. Notably, while $\mathrm{MoS}_{2}$ monolayer is a n-type semiconductor, phosphorene is $\mathrm{p}$ type semiconductor material. These results obtained for the libration frequencies of charged flake + substrate systems are interesting and herald that electrostatic charging can be utilized to tune the related dynamics.

Effect of Foreign Molecule. Here we show that the rotational dynamics of nanoflakes on the selected substrates can be modified by a foreign molecule anchored to the flake. Depending on the character of binding, the anchored molecule can modify the interaction between the flake and substrate. Chemical binding or chemisorption may result in significant and local charge rearrangements, which in turn causes changes in the angular force constant. Also depending on the size of the molecule, the moment of inertia relative to the rotation axis may also change with the change of adsorption site on the flake. As a proof of concept, we consider glycine, the simplest amino acid molecule, with chemical formula $\mathrm{C}_{2} \mathrm{H}_{5} \mathrm{NO}_{2}$, anchored to specific $\alpha$-PNFH and $\beta$-PNFH nanoflakes of type $\mathrm{t}-\mathrm{zz} / 33 \mathrm{H}+15 \mathrm{H}$. The results of our calculations are presented in Table 2. The molecular and structural configuration of glycine + flake systems on related substrates are given in Figure 4. We considered various initial configurations of glycine on flake near to its center, so that the binding configurations given in Figure 4 correspond to the most stable states with minimum energy. In this respect, glycine is always adsorbed on flake + substrate from $\mathrm{NH}_{2}$ tail forming a nearly perpendicular standing of $\mathrm{C}-\mathrm{N}$ bond on flake surface. It is seen that when a glycine molecule is anchored to flake + substrate system, the angular (or libration) frequency show a slight deviation within $\pm \approx 0.05 \times 10^{12} \mathrm{rad} / \mathrm{s}$ from the original, bare frequencies. For example, the angular frequencies of both $\alpha$-PNFH and $\beta$-PNFH on $\mathrm{MoS}_{2}$ surface decrease slightly upon the adsorption of glycine. On the other hand, in certain cases, the angular frequencies increase when glycine is adsorbed to the flake. For reasons pointed out in the beginning of this section, the position of the rotation axis may be subject to very minute translations in small angle rotations around the minimum energy configurations, unless a giant molecule is anchored to the flake. With fixed rotation axis, the angular frequency may change at most by $\sim 10 \%$ for glycine anchored at farthest possible site on the flake. Nevertheless, the change of frequency with the absorbed molecule may be taken as a fingerprint of the molecule and can be developed as a detector.

Effect of Electric Field. Next, we examine the effect of the external electric field, which was applied perpendicular to plane of the flake + substrate system. An applied electric field can lead to the transfer of electrons in the antiparallel direction of the field. In Table 2, the calculated angular force constant, $\kappa$ and angular frequency, $\omega$ values are presented for a representative flake, c-zz/24P $+12 \mathrm{H}$ of $\alpha$ - and $\beta$-PNFH on specific monolayer substrates. Upon an external electric field of $0.5 \mathrm{~V} / \AA ̊$, libration frequencies increase on graphene sheet. The perpendicular electric field can enhance the $\mathrm{p}_{z}$ orbital bonding of graphene yielding a relatively larger angular force constants and libration frequencies. However, frequencies decrease on phosphorene monolayer substrate for both $\alpha$ - and $\beta$-PNFH. The electric field affects the libration frequencies of $\alpha$ - and $\beta$ PNFHs on $\mathrm{MoS}_{2}$ surface in an opposite manner. These results suggest that the external electric field can be used to tune the rotational dynamics of flakes on monolayer substrates and hence to monitor nanomechanical devices.

Effect of Pressure. The effect of the pressure, or the external force applied perpendicular to the flake on the electronic structure is examined for $\beta$-PNFH flake of $\mathrm{t}-\mathrm{zz} / 33 \mathrm{P}$ $+15 \mathrm{H}$ type on $\beta$-P. The free-standing $\beta$-PNFH flake of $\mathrm{t}-\mathrm{zz} /$ 
$33 \mathrm{P}+15 \mathrm{H}$ type has a HOMO-LUMO gap of $E_{H-L}=2.62 \mathrm{eV}$. When placed on $\beta$-P monolayer, the band gap of the combined flake + monolayer with optimized equilibrium structure becomes $E_{g}=0.98 \mathrm{eV}$. The perpendicular compressive force is generated by reducing the equilibrium spacing $z_{o}=3.20 \AA$ between the flake and monolayer to $z=2.70 \AA$. This way, an average external force of $\sim 7 \mathrm{nN}$ is generated. As a result of this compressive force the band gap of the combined flake + substrate system narrows down to $E_{g}=0.53 \mathrm{eV}$. This is a considerable effect, which shows the efficient tunability of the band gap by perpendicular compressive force exerting on the flake. Such a force can be generated by the tip of an atomic force microscope. Alternatively, this physical event, as an example, can be utilized to estimate the force by measuring the band gap. In addition, reduction of the band gap under the perpendicular compressive force is generated by the increasing coupling between the flake and monolayer. Consequently, $\kappa$ and libration frequency can be changed by an applied force.

\section{TRANSLATIONAL AND ROTATIONAL DYNAMICS OF NANOFLAKES ON MONOLAYERS}

In this section, we examine the translational and large angle rotational dynamics. The energy barriers in the rotational and translational dynamics of nanoflakes on monolayer substrates are also crucial and pertain closely to the nearly frictionless rotation and sliding. ${ }^{40}$ Further to the variation of interaction energy between flake and substrate, $E_{i}(z, \theta)$ in the rotational dynamics as described in Figure 3, we also calculated the variation of the lowest interaction energy, $E_{i}(x, y, z)$, associated with the translation of phosphorene nanoflakes on selected monolayers.

Energy Barriers in Translational and Rotational Dynamics. In Figure 5, we display the calculated energy profile, $\left(E_{i}(x, y, z)\right)$ for a selected $\alpha$-PNFH and $\beta$-PNFH flake of c-zz/24P+12H type in the course of their translational motion on monolayer substrates, i.e. $\alpha$-PNFH + graphene, $\alpha$ $\mathrm{PNFH}+\alpha$-P, $\beta$-PNFH $+\beta$-P, and $\beta$-PNFH $+\mathrm{MoS}_{2}$. In these calculations the distance between the flake and substrate, $z$ is optimized for each lateral $(x, y)$ position to give highest attraction. There is a $103 \mathrm{meV}$ energy barrier for $\alpha$-PNFH on graphene between AA and unstable AC configurations. The corresponding translation of the flake between these two states are $\approx 4 \AA$. The $\mathrm{AB}$ state is a local minimum corresponding to further $\approx 5.5 \AA$ translation along zigzag direction. $\mathrm{AB}$ stacking of $\alpha$-PNFH on $\alpha$-P is a global minimum. $\beta$-PNFH has AA stacking on both $\beta$-P and $\mathrm{MoS}_{2}$ surfaces as global minimum state. In both cases, the $\mathrm{AB}$ state is global maximum with 329 and $256 \mathrm{meV}$ higher energies than the AA state on $\beta$-P and $\mathrm{MoS}_{2}$ surfaces, respectively. Upon further translations in the same direction from unstable global maxima in parts $b-d$ of Figure 5, one arrives at another global minimum.

Here we define the energy barriers involved in the translational dynamics as $Q_{T}\left(Q_{T}^{\prime}\right)$ corresponding to the energy difference between the global minimum and local minimum (global minimum and global maximum). In the course of sliding, a flake, which snaps to another minimum by going over these barriers generates phonons and dissipates mechanical energy. ${ }^{41,42}$ The form and height of the barrier energy have a close bearing on the sliding friction. The microscopic aspects of sliding friction and the energy dissipation related with it have been actively studied. ${ }^{41-43}$ The energy dissipation can be revealed from the hysteresis involved in the translational motion of the flake. It has been

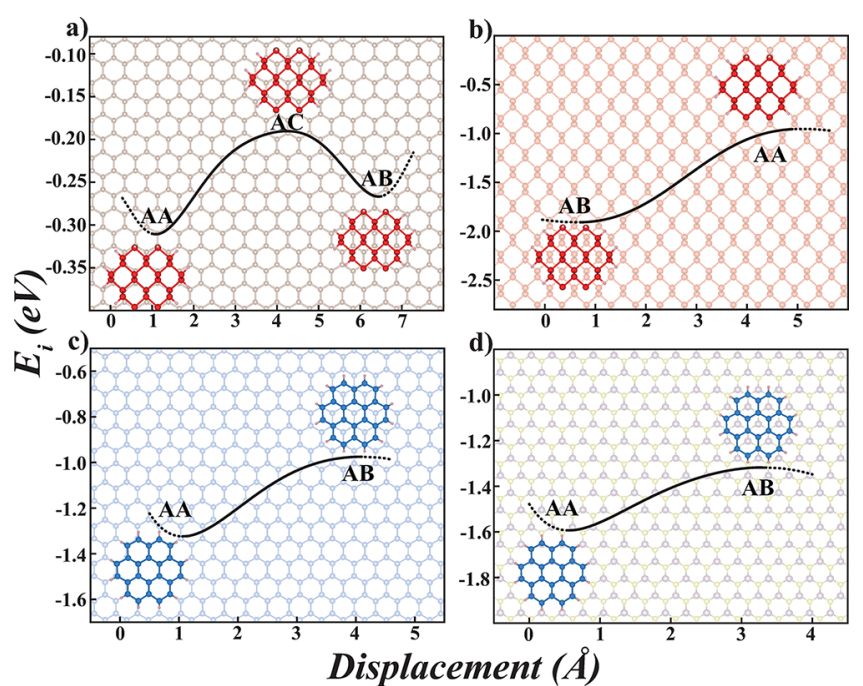

Figure 5. Variation of the energy in the translational dynamics of specific flakes on the selected monolayer substrates: Optimized interaction energy, $E_{i}(x, y)$ corresponding to a nanoflake displacing on the specific monolayers. The distance $z$ between the nanoflake and the monolayer is optimized for each value of $(x, y)$ point on the substrate monolayer. The selected flake type is c-zz/24P+12H. (a) $\alpha$ PNFH moving on graphene. (b) $\alpha$-PNFH moving on $\alpha$-P. (c) $\beta$ PNFH moving on $\beta$-P. (d) $\beta$-PNFH moving on $\mathrm{MoS}_{2}$ substrate. The path of translation starts from the global minimum configuration (or stacking), passes through the global maximum and ends either at a local minimum as shown in part a, or at the global maximum as shown in part $b$.

shown that, depending on the height and form of the barrier energy, $Q_{T}$, nearly frictionless sliding can be attained in the relative translational motion of selected monolayers. ${ }^{29,30,44}$

As discussed in the previous section, similar energy barriers can be defined for the rotational dynamics as $Q_{R}$ and $\left(Q_{R}^{\prime}\right)$, which correspond to the energy difference between global minimum and the local minimum (global minimum and global maximum). Beyond small angle vibrations with well-defined libration frequencies, the energy barriers, $Q_{R}$ and $Q_{R}^{\prime}$ in large angle or full rotation gain importance for the rotational friction, which may be critical in nanomechanics. Notably, the rotational and translational displacements can give rise to small changes in the electronic structure as discussed in the forthcoming section. Our extensive study on the profiles of optimized interaction energy between diverse nanoflakes executing rotational and translational motions on specific substrates and involved energy barriers thereof constitute a framework for further study of dynamics and energy dissipation. Our results are presented in Table 3.

The rotational $\left(Q_{R}\right.$ and $\left.Q_{R}^{\prime}\right)$ and translational $\left(Q_{T}\right.$ and $\left.Q_{T}^{\prime}\right)$ energy barriers for $\alpha$-PNFH on $\alpha$-P and $\beta$-PNFH on $\beta$-P, respectively, are significantly high as compared to the barriers on graphene sheet. This is in compliance with the fact that one usually attains high friction constants between the commensurate sliding surfaces. $Q_{R}$ values on graphene sheet are between 7 and $53 \mathrm{meV}$ for $\alpha$-PNFH and $3-432 \mathrm{meV}$ for $\beta$-PNFH. These local energy barriers are relatively low to excite these flakes to nearest local minimum on graphene, so that these excitations for various flake + substrate system can be realized at ambient temperature and above. $Q_{R}^{\prime}$ values, which are the energy barriers between global minimum and global maximum, increase with increasing flake size. However, $Q_{R}$ and $Q_{T}$ values 
Table 3. Calculated Optimized Energy Barriers (in meV) Involved in the Translational and Rotational Dynamics of $\alpha$-PNFH and $\beta$-PNFH on Various Monolayer Substrates ${ }^{a}$

\begin{tabular}{|c|c|c|c|c|c|c|c|c|}
\hline & \multicolumn{4}{|c|}{ graphene } & \multicolumn{4}{|c|}{$\alpha-\mathrm{P}$} \\
\hline & $\mathrm{Q}_{T}$ & $\mathrm{Q}_{T}^{\prime}$ & $\mathrm{Q}_{R}$ & $\mathrm{Q}_{R}^{\prime}$ & $\mathrm{Q}_{T}$ & $\mathrm{Q}_{T}^{\prime}$ & $\mathrm{Q}_{\mathrm{R}}$ & $\mathrm{Q}_{R}^{\prime}$ \\
\hline $\mathrm{t}-\mathrm{zz} / 13 \mathrm{P}+9 \mathrm{H}$ & 3 & 42 & 31 & 55 & 357 & - & 248 & 327 \\
\hline $\mathrm{t}-\mathrm{ac} / 18 \mathrm{P}+12 \mathrm{H}$ & 6 & 73 & 10 & 62 & 540 & - & 249 & 466 \\
\hline $\mathrm{t}-\mathrm{zz} / 22 \mathrm{P}+12 \mathrm{H}$ & 12 & 67 & 53 & 74 & 592 & - & 361 & 514 \\
\hline $\mathrm{c}-\mathrm{zz} / 24 \mathrm{P}+12 \mathrm{H}$ & 23 & 103 & 7 & 90 & 703 & - & 298 & 781 \\
\hline $\mathrm{p}-\mathrm{zz} / 28 \mathrm{P}+14 \mathrm{H}$ & 9 & 85 & 42 & 119 & 768 & - & 337 & 720 \\
\hline $\mathrm{t}-\mathrm{zz} / 33 \mathrm{P}+15 \mathrm{H}$ & 29 & 120 & 29 & 107 & 905 & - & 383 & 815 \\
\hline \multirow[t]{3}{*}{$\mathrm{t}-\mathrm{ac} / 36 \mathrm{P}+18 \mathrm{H}$} & 33 & 130 & 29 & 140 & 1043 & - & 470 & 938 \\
\hline & \multicolumn{4}{|c|}{ graphene } & \multicolumn{4}{|c|}{$\beta$-P } \\
\hline & $\mathrm{Q}_{T}$ & $\mathrm{Q}_{T}^{\prime}$ & $\mathrm{Q}_{R}$ & $\mathrm{Q}_{R}^{\prime}$ & $\mathrm{Q}_{T}$ & $\mathrm{Q}_{T}^{\prime}$ & $\mathrm{Q}_{R}$ & $\mathrm{Q}_{R}^{\prime}$ \\
\hline $\mathrm{t}-\mathrm{zz} / 13 \mathrm{P}+9 \mathrm{H}$ & 37 & - & 3 & 47 & 68 & 170 & 17 & 438 \\
\hline $\mathrm{t}-\mathrm{ac} / 18 \mathrm{P}+12 \mathrm{H}$ & 11 & - & 25 & 86 & 138 & 235 & 409 & 699 \\
\hline $\mathrm{t}-\mathrm{zz} / 22 \mathrm{P}+12 \mathrm{H}$ & 23 & - & 22 & 58 & 136 & 289 & 63 & 676 \\
\hline$c-z z / 24 \mathrm{P}+12 \mathrm{H}$ & 22 & - & 28 & 53 & 242 & 329 & 462 & 861 \\
\hline $\mathrm{p}-\mathrm{zz} / 28 \mathrm{P}+14 \mathrm{H}$ & 21 & - & 28 & 115 & 230 & 354 & 339 & 681 \\
\hline $\mathrm{t}-\mathrm{zz} / 33 \mathrm{P}+15 \mathrm{H}$ & 0.1 & - & 73 & 118 & 223 & 422 & 760 & 1234 \\
\hline \multirow[t]{3}{*}{$\mathrm{t}-\mathrm{ac} / 36 \mathrm{P}+18 \mathrm{H}$} & 3 & - & 432 & 722 & 321 & 472 & 739 & 1.274 \\
\hline & \multicolumn{4}{|c|}{$\mathrm{MoS}_{2}+\alpha-\mathrm{PNFH}$} & \multicolumn{4}{|c|}{$\mathrm{MoS}_{2}+\beta-\mathrm{PNFH}$} \\
\hline & $\mathrm{Q}_{T}$ & $\mathrm{Q}_{T}^{\prime}$ & $\overline{\mathrm{Q}_{R}}$ & $\mathrm{Q}_{R}^{\prime}$ & $\mathrm{Q}_{T}$ & $\overline{\mathrm{Q}_{T}^{\prime}}$ & $\mathrm{Q}_{R}$ & $\mathrm{Q}_{R}^{\prime}$ \\
\hline $\mathrm{c}-\mathrm{zz} / 24 \mathrm{P}+12 \mathrm{H}$ & 62 & 84 & 212 & 365 & 145 & 256 & 385 & 845 \\
\hline $\mathrm{t}-\mathrm{zz} / 33 \mathrm{P}+15 \mathrm{H}$ & 45 & 74 & 226 & 400 & 151 & 327 & 698 & 1170 \\
\hline
\end{tabular}

${ }^{a} \mathrm{Q}_{T}$ : energy difference between global minimum and local minimum in translational dynamics. $\mathrm{Q}_{T}^{\prime}$ : energy difference between global minimum and global maximum in translational dynamics. $\mathrm{Q}_{R}$ : energy difference between global minimum and local minimum in rotational dynamics. $\mathrm{Q}_{R}^{\prime}$ : energy difference between global minimum and global maximum in rotational dynamics.

Table 4. Change of Electronic Band Gap under 0, 10, 20, 30, and 40 Rotation and 0, 0.5, 1.0, 1.5, and 2.0 Å Linear Displacement (Translation) of $\alpha$ - and $\beta$-PNFH of Type t-zz/33P+15H on Various Substrates ${ }^{a}$

\begin{tabular}{|c|c|c|c|c|c|c|c|c|c|}
\hline \multicolumn{5}{|c|}{ rotation } & \multicolumn{5}{|c|}{ translation } \\
\hline \multirow[b]{2}{*}{ rotation $(\mathrm{deg})$} & \multicolumn{2}{|c|}{$\beta$-PNFH } & \multicolumn{2}{|c|}{$\alpha$-PNFH } & \multirow[b]{2}{*}{ disp. $(\AA ̊)$} & \multicolumn{2}{|c|}{$\beta$-PNFH } & \multicolumn{2}{|c|}{$\alpha$-PNFH } \\
\hline & subs. & $E_{g}(\mathrm{meV})$ & subs. & $E_{g}(\mathrm{meV})$ & & subs. & $E_{g}(\mathrm{meV})$ & subs. & $E_{g}(\mathrm{meV})$ \\
\hline \multirow[t]{2}{*}{0} & $\beta$-P & 981 & $\alpha-\mathrm{P}$ & 850 & 0 & $\beta-\mathrm{P}$ & 981 & $\alpha-\mathrm{P}$ & 850 \\
\hline & $\mathrm{MoS}_{2}$ & 1249 & $\mathrm{MoS}_{2}$ & 1298 & & $\mathrm{MoS}_{2}$ & 1249 & $\mathrm{MoS}_{2}$ & 1298 \\
\hline \multirow[t]{2}{*}{10} & $\beta$-P & 1149 & $\alpha-\mathrm{P}$ & 875 & 0.5 & $\beta$-P & 999 & $\alpha-\mathrm{P}$ & 876 \\
\hline & $\mathrm{MoS}_{2}$ & 1294 & $\mathrm{MoS}_{2}$ & 1306 & & $\mathrm{MoS}_{2}$ & 1244 & $\mathrm{MoS}_{2}$ & 1297 \\
\hline \multirow[t]{2}{*}{20} & $\beta$-P & 1152 & $\alpha-\mathrm{P}$ & 875 & 1 & $\beta$-P & 1030 & $\alpha-\mathrm{P}$ & 890 \\
\hline & $\mathrm{MoS}_{2}$ & 1326 & $\mathrm{MoS}_{2}$ & 1315 & & $\mathrm{MoS}_{2}$ & 1269 & $\mathrm{MoS}_{2}$ & 1339 \\
\hline \multirow[t]{2}{*}{30} & $\beta$-P & 1180 & $\alpha-\mathrm{P}$ & 870 & 1.5 & $\beta$-P & 1037 & $\alpha-\mathrm{P}$ & 838 \\
\hline & $\mathrm{MoS}_{2}$ & 1332 & $\mathrm{MoS}_{2}$ & 1321 & & $\mathrm{MoS}_{2}$ & 1262 & $\mathrm{MoS}_{2}$ & 1347 \\
\hline \multirow[t]{2}{*}{40} & $\beta$-P & 1149 & $\alpha-\mathrm{P}$ & 875 & 2 & $\beta$-P & 993 & $\alpha-\mathrm{P}$ & 836 \\
\hline & $\mathrm{MoS}_{2}$ & 1325 & $\mathrm{MoS}_{2}$ & 1310 & & $\mathrm{MoS}_{2}$ & 1242 & $\mathrm{MoS}_{2}$ & 1315 \\
\hline
\end{tabular}

${ }^{a_{\text {The }}}$ motion starts from global minimum state.

exhibit rather irregular behavior. Both $Q_{R}$ and $Q_{R}^{\prime}$ values for $\alpha$ and $\beta$-PNFHs follow a trend on the surfaces: graphene $<\mathrm{MoS}_{2}$ $<$ phosphorene. It is clear that $\alpha$-PNFH on $\alpha$-P and $\beta$-PNFH on $\beta$-P cannot be easily excited to another metastable state due to their equilibrium configurations settled in deep valleys on energy surface. It is also seen that both rotational and translational energy barrier values depend mainly on whether the flake structures are commensurate or incommensurate to the substrate monolayers rather than flake size.

In Figure 3, we display the variation of the interaction energy, $E_{i}(z, \theta)$ of $\alpha$ - and $\beta$-PNFH of type c-zz $/ 24 \mathrm{P}+12 \mathrm{H}$ on monolayer substrates as a function of the angular displacement, $\theta$, around the equilibrium. Variation of angular force constants, $\kappa$ and torque, $\tau=-\partial E / \partial \theta$, as a function of $\theta$ are also given in this figure. It is seen that both $\kappa$ and $\tau$ display an oscillatory behavior with varying periods. Notably, $E_{i}$ is optimized relative to the height of the flake above the substrate, $z$ for each $\theta$. $\alpha$ $\mathrm{PNFH}$ on graphene exhibits a rather symmetric behavior due to rotation around global minimum on graphene. $\alpha$-PNFH can attain also a local minimum, which has only $7 \mathrm{meV}$ higher energy than equilibrium state, on graphene surface upon a $\pi / 3$ counterclockwise rotation from $\mathrm{AA}$ to $\mathrm{AA}^{\prime}$ stacking. But the required energy to overcome the barrier is $\approx 90 \mathrm{meV}$ from $\mathrm{AA}$ to $\mathrm{AA}^{\prime \prime}$ configuration. There is also another local minimum available at $\pm \pi / 6$ rotation of $\alpha$-PNFH on graphene. $\alpha$-PNFH has a distinct global minimum on $\alpha$-P surface surrounded by high energy barriers. In that case, $\alpha$-PNFH has to overcome an $781 \mathrm{meV}$ energy barrier to reach a local minimum which is located about $\approx \pi / 4$ clockwise rotation. Another local minimum, $\mathrm{AA}^{\prime}$, is located at $\pm \approx 0.4 \pi$. The energy profile of 
$\beta$-PNFH displays rather symmetrical behavior on $\beta$-P and $\mathrm{MoS}_{2}$ surfaces. In both cases, the structures with commensurate hexagonal rings of flake and substrate corresponds to global minimum state with $\mathrm{AA}$ stacking, while $\mathrm{AA}^{\prime \prime}$ stacking yields a global maximum state with remarkable contribution to electrostatic energy by incommensurate atoms.

\section{ELECTRONIC STRUCTURE}

Further to dynamical parameters between nanoflake and monolayers discussed above, the electronic structures are expected to vary depending on their relative configurations. However, the variation of the energy band gaps of the flake + monolayer is small due to the weak coupling between them. In Table 4, we summarized the variation of the energy gap of $\alpha$ $\mathrm{PNFH}$ and $\beta$-PNFH nanoflakes of type $\mathrm{t}-\mathrm{zz} / 33 \mathrm{P}+15 \mathrm{H}$ on $\alpha-\mathrm{P}$, $\beta$-P and $\mathrm{MoS}_{2}$ monolayers with angular displacement $\theta$. It is seen that the variation of band gap with $\theta$ is generally small, but is significant for $\beta$-PNFH on $\beta$-P $(200 \mathrm{meV})$ and on $\mathrm{MoS}_{2}$ $(83 \mathrm{meV})$ monolayer.

Furthermore, we also examined the variation of electronic band gap with linear displacement of the same flake ( $\mathrm{t}-\mathrm{zz} / 33 \mathrm{P}$ $+15 \mathrm{H})$ on $\alpha$-P, $\beta$-P and $\mathrm{MoS}_{2}$ monolayers. The results are given in Table 4 . The band gap of $\beta$-PNFH on $\beta$-P changes up to $\approx 50 \mathrm{meV}$ by translation between two adjacent global minima. The related change of the same flake on $\mathrm{MoS}_{2}$ is relatively lower. However, band gap change of $\alpha$-PNFH on $\alpha$-P is low, while that of $\alpha$-PNFH on $\mathrm{MoS}_{2}$ goes up to $\approx 50 \mathrm{meV}$. It can be concluded that the band gap of flake+substrate composite system can be slightly modified by rotational or translational motion of flake relative to substrate.

We note that each nanoflake + monolayer system can be considered as a heterostructure consisting of a monolayer with 2D band structure and a molecule with discrete energy levels and HOMO-LUMO gap. Depending on the constituent elements this construction can be viewed as metal/semiconductor or semiconductor/semiconductor junction. For a junction like $\alpha$-PNFH or $\beta$-PNFH + graphene a Schottky barrier can be generated, which can be tuned by the external electric field. Similarly, for a heterostructure $\beta$-PNFH $+\beta$-P the electronic charge between the constituent elements and hence combined band gap can be tuned with the external electric field. Moreover, as discussed in previous sections, the band gap of heterostructure can be modified by the applied compressive force. Once the contacts to the constituent elements are set, these heterostructures may offer several device parameters.

\section{DISCUSSIONS AND CONCLUSIONS}

In this study we explored the rotational and translational dynamics of black and blue phosphorene flakes of diverse size and geometry on monolayer substrates like graphene, phosphorene and $\mathrm{MoS}_{2}$. The attractive interaction between the nanoflakes and substrates considered in this study is generally weak. Under this weak attraction, a phosphorene flake can execute simple harmonic motion relative to the monolayer substrate if it is displaced from the equilibrium position by small angles. The frequency of these vibration are called as libration frequency, which depends on the size, geometry of the flake and on the underlying subsrate monolayer. In this respect, it can be measured and constitutes a fingerprint for the flake. Moreover, the libration frequency can be modified by charging, external electric field and by external, compressive force. It changes also if a molecule is anchored on the flake. In this respect, small angle vibrations of flakes offer new alternative for detectors and devices.

When viewed as a heterostructure, nanoflake + monolayer junctions display combined electronic structure, which can be tuned with the rotation angle. The band gap of the semiconductor/semiconductor junction and the Schottky barrier of the metal(graphene)/semiconductor junction can be tuned by the applied external electric field. The combined electronic structure can also vary depending on the different equilibrium positions in the course of translational motion. Additionally, we showed that the pressure between the flake and substrate can be an external agent to monitor the electronic structure and dynamical properties.

The profiles of the interaction energy and the energy barriers thereof in the full rotation or translation of nanoflakes are of particular importance. We show that the energy barriers in the majority of cases studied here are rather low and have close bearings on the rotational and sliding friction. In this paper we calculate translational and rotational energy barriers of phosphorene nanoflakes on selected monolayers. While these energy barriers nanoflake and substrate specific, they are usually low and keep the promise of nearly frictionless rotation and sliding in nanomechanics.

In conclusion, the dynamics of phosphorene nanoflakes on selected monolayers, like graphene, black and blue phosphorene, and $\mathrm{MoS}_{2}$, considered in this paper constitute crucial and novel combined systems with dynamical and electronic parameters to be exploited in diverse types of detectors and junction devices. Also, a mesh of nanoflakes placed on a specific monolayer or a stack of multiple nanoflakes on a monolayer constitutes composite structures, which can offer novel physical properties to be used in mechanical, optical and electronic applications.

\section{AUTHOR INFORMATION}

\section{Corresponding Authors}

*(E.A.) E-mail: ethem.akturk@adu.edu.tr. Telephone: +902562130835-189. Fax: +902562135379.

*(S.C.) E-mail: ciraci@fen.bilkent.edu.tr. Telephone: +903122901216. Fax: +903122664579.

ORCID $\odot$

E. Aktürk: 0000-0002-1615-7841

Notes

The authors declare no competing financial interest.

\section{ACKNOWLEDGMENTS}

The computational resources are provided by TÜBİTAK ULAKBIM, High Performance and Grid Computing Center (TR-Grid e-Infrastructure).This research was supported by the TÜBITAK under Project No. 116F059. S.C. acknowledges financial support from the Academy of Sciences of Turkey TÜBA.

\section{REFERENCES}

(1) Geim, A. K.; Grigorieva, I. V. van der Waals heterostructures. Nature 2013, 499, 419-425.

(2) Li, C.; Gao, J.; Zi, Y.; Wang, F.; Niu, C.; Cho, J.-H.; Jia, Y. Asymmetric quantum confinement-induced energetically and spatially splitting Dirac rings in graphene/phosphorene/graphene heterostructure. Carbon 2018, 140, 164-170.

(3) Ponomarenko, L. A.; Geim, A. K.; Zhukov, A. A.; Jalil, R.; Morozov, S. V.; Novoselov, K. S.; Grigorieva, I. V.; Hill, E. H.; Cheianov, V. V.; Fal'ko, V. I.; et al. Tunable metal-insulator 
transition in double-layer graphene heterostructures. Nat. Phys. 2011, 7, 958-961.

(4) Britnell, L.; Gorbachev, R. V.; Jalil, R.; Belle, B. D.; Schedin, F.; Mishchenko, A.; Georgiou, T.; Katsnelson, M. I.; Eaves, L.; Morozov, S. V.; et al. Field-Effect Tunnelling Transistor Based on Vertical Graphene Heterostructures. Science 2012, 335, 947-950.

(5) Haigh, S. J.; Gholinia, A.; Jalil, R.; Romani, S.; Britnell, L.; Elias, D. C.; Novoselov, K. S.; Ponomarenko, L. A.; Geim, A. K.; Gorbachev, R. Cross-sectional imaging of individual layers and buried interfaces of graphene-based heterostructures and superlattices. Nat. Mater. 2012, 11, 764-767.

(6) Dean, C.; Young, A. F.; Wang, L.; Meric, I.; Lee, G.-H.; Watanabe, K.; Taniguchi, T.; Shepard, K.; Kim, P.; Hone, J. Graphene based heterostructures. Solid State Commun. 2012, 152, 1275-1282.

(7) Gorbachev, R. V.; Geim, A. K.; Katsnelson, M. I.; Novoselov, K. S.; Tudorovskiy, T.; Grigorieva, I. V.; MacDonald, A. H.; Morozov, S. V.; Watanabe, K.; Taniguchi, T.; et al. Strong Coulomb drag and broken symmetry in double-layer graphene. Nat. Phys. 2012, 8, 896901.

(8) Georgiou, T.; Jalil, R.; Belle, B. D.; Britnell, L.; Gorbachev, R. V.; Morozov, S. V.; Kim, Y.-J.; Gholinia, A.; Haigh, S. J.; Makarovsky, O.; et al. Vertical field-effect transistor based on graphene-WS2 heterostructures for flexible and transparent electronics. Nat. Nanotechnol. 2013, 8, 100-103.

(9) Yoon, J.; Park, W.; Bae, G. Y.; Kim, Y.; Jang, H. S.; Hyun, Y.; Lim, S. K.; Kahng, Y. H.; Hong, W. K.; Lee, B. H.; et al. Highly Flexible and Transparent Multilayer MoS2 Transistors with Graphene Electrodes. Small 2013, 9, 3295-3300.

(10) Roy, T.; Tosun, M.; Kang, J. S.; Sachid, A. B.; Desai, S. B.; Hettick, M.; Hu, C. C.; Javey, A. Field-Effect Transistors Built from All Two-Dimensional Material Components. ACS Nano 2014, 8, 6259-6264.

(11) Aras, M.; Kilic, C.; Ciraci, S. Planar heterostructures of singlelayer transition metal dichalcogenides: Composite structures, Schottky junctions, tunneling barriers, and half metals. Phys. Rev. B: Condens. Matter Mater. Phys. 2017, 95, 075434.

(12) Aras, M.; Kilic, C.; Ciraci, S. Lateral and Vertical Heterostructures of Transition Metal Dichalcogenides. J. Phys. Chem. C 2018, 122, 1547-1555.

(13) Gao, G.; Gao, W.; Cannuccia, E.; Taha-Tijerina, J.; Balicas, L.; Mathkar, A.; Narayanan, T. N.; Liu, Z.; Gupta, B. K.; Peng, J.; et al. Artificially Stacked Atomic Layers: Toward New van der Waals Solids. Nano Lett. 2012, 12, 3518-3525.

(14) Yankowitz, M.; Jung, J.; Laksono, E.; Leconte, N.; Chittari, B. L.; Watanabe, K.; Taniguchi, T.; Adam, S.; Graf, D.; Dean, C. R. Dynamic band-structure tuning of graphene moire superlattices with pressure. Nature 2018, 557, 404-408.

(15) Georgakilas, V.; Otyepka, M.; Bourlinos, A. B.; Chandra, V.; Kim, N.; Kemp, K. C.; Hobza, P.; Zboril, R.; Kim, K. S. Functionalization of Graphene: Covalent and Non-Covalent Approaches, Derivatives and Applications. Chem. Rev. 2012, 112, 6156-6214.

(16) Lazar, P.; Karlický, F.; Jurečka, P.; Kocman, M.; Otyepková, E.; Šafárová, K.; Otyepka, M. Adsorption of Small Organic Molecules on Graphene. J. Am. Chem. Soc. 2013, 135, 6372-6377.

(17) Bueno, R. A.; Martínez, J. I.; Luccas, R. F.; del Árbol, N. R.; Munuera, C.; Palacio, I.; Palomares, F. J.; Lauwaet, K.; Thakur, S.; Baranowski, J. M.; et al. Highly selective covalent organic functionalization of epitaxial graphene. Nat. Commun. 2017, 8, 15306.

(18) Lyalin, A.; Nakayama, A.; Uosaki, K.; Taketsugu, T. Functionalization of Monolayer h-BN by a Metal Support for the Oxygen Reduction Reaction. J. Phys. Chem. C 2013, 117, 2135921370.

(19) Weng, Q.; Wang, X.; Wang, X.; Bando, Y.; Golberg, D. Functionalized hexagonal boron nitride nanomaterials: emerging properties and applications. Chem. Soc. Rev. 2016, 45, 3989-4012.

(20) Huang, Y. L.; Zheng, Y. J.; Song, Z.; Chi, D.; Wee, A. T. S.; Quek, S. Y. The organic-2D transition metal dichalcogenide heterointerface. Chem. Soc. Rev. 2018, 47, 3241-3264.
(21) Sarkar, D.; Xie, X.; Kang, J.; Zhang, H.; Liu, W.; Navarrete, J.; Moskovits, M.; Banerjee, K. Functionalization of Transition Metal Dichalcogenides with Metallic Nanoparticles: Implications for Doping and Gas-Sensing. Nano Lett. 2015, 15, 2852-2862.

(22) Shirai, Y.; Osgood, A. J.; Zhao, Y.; Yao, Y.; Saudan, L.; Yang, H.; Yu-Hung, C.; Alemany, L. B.; Sasaki, T.; Morin, J. F.; et al. Surface-Rolling Molecules. J. Am. Chem. Soc. 2006, 128, 4854-4864.

(23) Van Delden, R. A.; ter Wiel, M. K. J.; Pollard, M. M.; Vicario, J.; Koumura, N.; Feringa, B. L. Unidirectional molecular motor on a gold surface. Nature 2005, 437, 1337-1340.

(24) Peymanirad, F.; Singh, S. K.; Ghorbanfekr-Kalashami, H.; Novoselov, K. S.; Peeters, F. M.; Neek-Amal, M. Thermal activated rotation of graphene flake on graphene. 2D Mater. 2017, 4, 025015.

(25) Akturk, O. U.; Akturk, E.; Gurel, H. H.; Ciraci, S. Tunable dynamics of a flake on graphene: Libration frequency. Phys. Rev. B: Condens. Matter Mater. Phys. 2017, 95, 125413.

(26) Wang, D.; Chen, G.; Li, C.; Cheng, M.; Yang, W.; Wu, S.; Xie, G.; Zhang, J.; Zhao, J.; Lu, X.; et al. Thermally Induced Graphene Rotation on Hexagonal Boron Nitride. Phys. Rev. Lett. 2016, 116, 126101.

(27) Choe, j.; Lee, Y.; Fang, L.; Lee, G. D.; Bao, Z.; Kim, K.; et al. Direct imaging of rotating molecules anchored on graphene. Nanoscale 2016, 8, 13174-13180.

(28) Lebedeva, I. V.; Knizhnik, A. A.; Popov, A. M.; Ershova, O. V.; Lozovik, Y. E.; Potapkin, B. V. Fast diffusion of a graphene flake on a graphene layer. Phys. Rev. B: Condens. Matter Mater. Phys. 2010, 82, 155460 .

(29) Cahangirov, S.; Ataca, C.; Topsakal, M.; Sahin, H.; Ciraci, S. Frictional Figures of Merit for Single Layered Nanostructures. Phys. Rev. Lett. 2012, 108, 126103.

(30) Cahangirov, S.; Ciraci, S.; Ozcelik, V. O. Superlubricity through graphene multilayers between $\mathrm{Ni}(111)$ surfaces. Phys. Rev. B: Condens. Matter Mater. Phys. 2013, 87, 205428.

(31) Sorkin, V.; Zhang, Y. W. Rotation-dependent epitaxial relations between graphene and the Si-terminated SiC substrate. Phys. Rev. B: Condens. Matter Mater. Phys. 2010, 82, 085434.

(32) Schaffert, J.; Cottin, M. C.; Sonntag, A.; Karacuban, H.; Bobisch, C. A.; Lorente, N.; Gauyacq, J. P.; Möller, R. Imaging the dynamics of individually adsorbed molecules. Nat. Mater. 2013, 12, 223-227.

(33) Ishikawa, T.; Hayes, S. A.; Keskin, S.; Corthey, G.; Hada, M.; Pichugin, K.; Marx, A.; Hirscht, J.; Shionuma, K.; Onda, K.; et al. Direct observation of collective modes coupled to molecular orbitaldriven charge transfer. Science 2015, 350, 1501-1505.

(34) Kresse, G.; Furthmüller, J. Efficient iterative schemes for ab initio total-energy calculations using a plane-wave basis set. Phys. Rev. B: Condens. Matter Mater. Phys. 1996, 54, 11169-11186.

(35) Kresse, G.; Furthmüller, J. Efficiency of ab-initio total energy calculations for metals and semiconductors using a plane-wave basis set. Comput. Mater. Sci. 1996, 6, 15-50.

(36) Perdew, J. P.; Burke, K.; Ernzerhof, M. Generalized Gradient Approximation Made Simple. Phys. Rev. Lett. 1996, 77, 3865-3868.

(37) Grimme, S. Semiempirical GGA-type density functional constructed with a long-range dispersion correction. J. Comput. Chem. 2006, 27, 1787-1799.

(38) Soler, J. M.; Artacho, E.; Gale, J. D.; García, A.; Junquera, J.; Ordejón, P.; Sánchez-Portal, D. The SIESTA method for ab-initio order-N materials simulation. J. Phys.: Condens. Matter 2002, 14, 2745.

(39) Shi, Y.; Wang, L. Colletive vibrational spectraof $\alpha$ - and $\gamma$ glycine studied by terahertz and Raman spectroscopy. J. Phys. D: Appl. Phys. 2005, 38, 3741.

(40) Erdemir, A.; Eryilmaz, O. L.; Fenske, G. Synthesis of diamondlike carbon films with superlow friction and wear properties. J. Vac. Sci. Technol., A 2000, 18, 1987-1992.

(41) Zhong, W.; Tomanek, D. First-principles theory of atomic-scale friction. Phys. Rev. Lett. 1990, 64, 3054.

(42) Dag, S.; Ciraci, S. Atomic scale study of superlow friction between hydrogenated diamond surfaces. Phys. Rev. B: Condens. Matter Mater. Phys. 2004, 70, 241401. 
(43) Meyer, E.; Overney, R. O.; et al. Friction and wear of Langmuir-Blodgett films observed by friction force microscopy. Phys. Rev. Lett. 1992, 69, 1777.

(44) Dienwiebel, M.; Verhoeven, G. S.; Pradeep, N.; Frenken, J. W. M.; Heimberg, J. A.; Zandbergen, H. W. Superlubricity of Graphite. Phys. Rev. Lett. 2004, 92, 126101. 